\title{
DIRE DANS LE MONDE
}

\author{
Jocelyn BENOIST \\ Université Paris 1 Panthéon-Sorbonne \\ Institut des Sciences Juridique et Philosophique de la Sorbonne
}

Dans Dire le monde ${ }^{1}$, Francis Wolff avance de façon répétée la formule suivant laquelle le langage «fait monde $»^{2}$. Une telle expression peut être prise en plusieurs sens. On peut l'entendre d'abord au sens où le langage en lui-même constituerait un monde et, en effet, en un sens, lorsqu'il explicite et justifie cette formule, Francis nous montre que le langage partage quelque chose avec la notion de «monde " : sans doute en premier lieu son universalité ( $D M$, p. 19), car comme le monde est tout ce qui existe, et peut-être plus généralement tout ce qui est, de la même façon, le langage fondamentalement se définit comme ce qui peut parler de tout.

Il est peut-être moins immédiatement évident que le langage, comme le soutient également Francis, partage avec le monde une certaine forme de réflexivité. En ce point, on peut avoir le sentiment que c'est plutôt le langage qui prête un trait au monde que l'inverse. En effet, si, dans une tradition française qui certainement compte pour Francis, à savoir celle qui vient de Benveniste, il est caractéristique du langage qu'il puisse parler aussi et au premier chef de lui-même, ce en quoi le monde porterait en luimême une forme de réflexivité intrinsèque, ce en quoi constitutivement il pourrait se rapporter à lui-même est, dans une certaine entente de la notion de monde au moins, moins clair. En fait, le simple fait que Francis introduise cette idée indique déjà que son concept de «monde » ne peut se réduire à un concept cosmologique mais qu'il porte en lui quelque parenté avec celui mis en avant par Heidegger (si étrangère, en général, puisse lui être cette référence). Ce qui est en jeu ici, c'est le monde comme ce dans quoi je suis ou en tout cas un certain type d'être est : un être qui, précisément, peut parler. À ce prix, la réflexivité peut en effet revenir au monde.

De ce point de vue, on pouvait dire que, si le langage a quelque chose de fondamentalement mondain, le monde a son tour est en un sens essentiellement

\footnotetext{
${ }^{1}$ Francis Wolff, Dire le monde, Paris, P.U.F., 1997, cité dans l'édition Quadrige de 2004. Désormais DM.

${ }^{2}$ Dès la p. 20 (qui est la p. 2 de l' « Introduction » générale) dans l'édition Quadrige de 2004. Le motif est repris dans la préface de 2004 p. 9.
} 
linguistique : il lui est essentiel d'être traversé par langage. Et en effet c'est bien la thèse qu'articule essentiellement le livre de Francis : il n'y aurait pas de monde sans langage.

Cet énoncé, suivant un risque qu'entreprend de prévenir la préface à l'édition Quadrige de 2004, peut donner le sentiment de tomber dans l'idéalisme linguistique pur et simple. Comment le monde pourrait-il résulter de notre activité linguistique ?

Pour corriger l'impression de paradoxe que peut susciter une telle affirmation, l'auteur de Dire le monde prend soin d'introduire une distinction essentielle, qui supporte l'ensemble de l'ouvrage. Il nous invite à faire une différence entre le monde d'un coté et le réel de l'autre. S'il y a lieu pour un «faire monde», c'est que précisément, par lui-même, le réel n'est pas un monde. Il faut le transformer en monde et c'est le langage qui est l'auteur ou le vecteur - suivant une ambiguïté peut-être constitutive de cet ouvrage - de cette transformation.

En fait, une fois reformulée dans ces termes, la thèse a indubitablement une résonance kantienne. Et, à plus d'un titre, il n'est pas injuste de dire que la philosophie présentée dans Dire Le Monde s'identifie à une forme de kantisme linguisticisé.

Une telle transposition ne va pas absolument de soi. Elle soulève nécessairement la difficile question de la priorité du langage ou de l'esprit. De ce point de vue, la démarche déployée par l'auteur de Dire le monde choisit. Elle reverse résolument au compte du langage ce dont le kantisme traditionnel trouvait le principe dans l'esprit. En même temps, ce n'est possible que parce que Francis repère dans le langage des dimensions différentes, dont l'une réassume les engagements conscients de la subjectivité. Lorsqu'il est dit dans Dire Le Monde que le langage « fait monde », ce n'est pas la seule langue qui «fait monde », et l'analyse mise en œuvre se tient loin du relativisme linguistique qui est essentiellement relativisme à la langue ou en tout cas aux langues dans leur particularité. Le langage tel que l'entend Francis est aussi et en un sens d'abord, en tout cas de façon irréductible, parole et, comme l'articule de façon tranchée le deuxième temps du livre, tout autant que le langage dans son anonymat « fait monde », la parole le fait. En toute rigueur pour avoir un monde tel que l'entend Francis, il faut de façon indissociable que langue et parole contribuent à son déploiement. Ce monde, en effet, est essentiellement un monde dans lequel il y a des personnes et ces personnes y sont présentes d'abord sous la figure de pronoms personnels.

Dire que le langage fait monde, ce n'est donc pas priver ce monde de subjectivité mais bien au contraire, en un certain sens, y inscrire de façon essentielle la position des sujets, le bénéfice de l'analyse de Francis étant de faire apparaître cette 
position dans sa pluralité constitutive suivant celle des différents pronoms personnels ce dernier point faisant probablement en un sens défaut dans l'analyse kantienne. Il n'en reste pas moins qu'à aucun moment la nouvelle puissance accordée au langage ne menace le statut fondateur du Je. Elle en dégage simplement la véritable fonction, à travers la figure du locuteur ${ }^{3}$.

Il se pourrait néanmoins que ce passage sur le terrain la philosophie du langage ne soit pas sans conséquence par rapport à l'évaluation à donner de la thèse kantienne qu'on peut appeler thèse criticiste.

En toute fidélité à Kant, il s'agirait, semble-t-il, de distinguer ce que sont les choses en soi et ce qu'elles sont pour nous. Si l'on adopte le nouveau cadre d'analyse envisagé, on obtient donc les choses indépendamment du fait qu'on en parle d'un côté, et les choses en tant qu'on en parle de l'autre. En bon kantien, par rapport aux premières, Francis se déclare agnostique. De ces choses en elles-mêmes, par définition, nous ne pouvons rien dire ni savoir. Ce mystère ne mettrait néanmoins pas en péril l'objectivité puisque tout ce qui compte, à ce niveau, c'est que nous soyons capables de déterminer correctement ces choses dont nous parlons et que, au moyen des mots, semble-t-il, nous pensons.

Il y a pourtant lieu de s'interroger sur cette restriction kantienne. Qu'il y ait beaucoup de choses dont nous ne parlons pas et ne parlerons jamais, et pour lesquelles il est probable qu'en l'état actuel de notre langage nous n'avons pas de mots, est assez évident. Même si, en ce qui concerne le dernier point, on peut se demander si, avec un langage donné, pour peu qu'il offre des ressources syntaxiques suffisantes, il n'est pas toujours possible de parler de quoi que ce soit, quelle que soit la pauvreté apparente du vocabulaire disponible. Cependant le motif criticiste mis en avant par Francis, selon toute probabilité, ne porte pas tant sur ces choses qui restent et resteront peut-être à jamais en dehors du plan de ce qu'il nous aura été donné d'énoncer, que sur ces choses mêmes que nous énonçons. C'est par rapport à ces dernières choses que naît le soupçon qu'il pourrait y avoir un écart entre leur être et précisément ce que nous en disons. Cela non pas au sens où nous nous tromperions alors à leur endroit et donc ou ce que nous en dirions serait faux. Un autre énoncé alors aurait à corriger cette erreur première. Mais plutôt au sens où ce serait constitutivement qu'il y aurait une différence entre la chose

\footnotetext{
${ }^{3} \mathrm{C}$ 'est en ce sens que « l'allocution transcendantale » constitue un démarquage assez direct - et, d'une façon que ne démentirait d'ailleurs peut-être en un sens pas Kant ( $c f$. l'essai remarquable de Raphaël Ehrsam, Le problème du langage chez Kant, Paris, Vrin, coll. : «Bibliothèque d'histoire de la philosophie », 2016), la « vérité »-de « l'aperception transcendantale ». Cf. DM, p. 205.
} 
dite, que ce qu'on en dit soit vrai ou faux, et « la chose même » - c'est-à-dire la chose dans sa réalité.

À ce niveau s'impose une objection ou, tout au moins, une question : certes, il y a lieu de distinguer la chose et la chose dite, si, comme dans le langage ordinaire, on entend par là tout simplement ce qui est dit et en règle générale - peut-être pas toujours - l'est d'une chose. Mais précisément, là où nous parlons de la chose, c'est de la chose purement et simplement, et non de la chose en tant qu'elle est dite. Sur elle nous nous trompons ou nous sommes dans le vrai, mais c'est bien toujours d'elle et d'elle-même et certainement d'aucun double qui serait la «chose parlée » - qu'il y va dans nos vérités comme dans nos erreurs.

De ce point de vue, un malentendu sans doute grève l'histoire du représentationalisme. Celui-ci a fait du langage le modèle de l'esprit: il a interprété notre vie mentale comme faite d'autant de mots qui s'intercaleraient pour ainsi dire entre notre pensée et les choses. Cependant, ce faisant, il s'est inspiré d'un modèle erroné du langage. En effet, au moyen de ses mots, le locuteur se rapporte directement aux choses. Il n'en parle pas en tant que parlées ; il en parle tout court.

Évidemment, il est toujours possible de rapporter une telle conviction à une forme de naïveté, d'en faire une illusion métaphysique de principe ${ }^{4}$. Notre foi naturelle en la transparence du langage suffit-elle à le rendre transparent? Trop souvent nous croyons savoir parfaitement de quoi nous parlons sans en réalité que la référence de nos mots soit bien claire - ni même qu'il soit clair qu'ils en aient aucune.

Un tel doute cependant porte sur l'usage que nous avons alors de nos mots et non sur ces mots eux-mêmes. Le problème n'est pas que les mots ne réfèrent pas, ou bien mal, à quelque chose qui n'aurait de sens que comme leur propre référence, mais plutôt que, en l'occurrence, nous ne nous sommes pas donné les moyens ou n'avons pas fait l'effort de réellement référer. Il se pourrait que de ce point de vue le cas de la référence obscure ou incomplète constitue une fausse fenêtre. Là où on réfère à moitié, en réalité, en un certain sens, on ne réfère pas du tout, précisément parce que référer veut dire définir, au moyen des mots, une prise déterminée sur une certaine réalité - une prise telle qu'on puisse dire des choses vraies ou fausses de cette réalité même.

La transparence linguistique, de ce point de vue, n'a rien d'un droit naturel. Elle est un acquis, le résultat d'un effort du sujet s'appropriant à chaque fois un outil collectif aux fins de l'expression non moins collective - par construction - de quelque

${ }^{4}$ Sur cette « illusion », voir DM, p. 16, n. 2. 
chose qui peut être absolument personnel. Mais ce ne sont pas les mots qui sont opaques, c'est nous qui sommes opaques à nous-mêmes tant que nous n'avons pas réussi à donner une forme explicite à ce que nous pensons.

Le critère de la transparence acquise est précisément que l'on voit clairement la différence que ce que nous disons fait dans le réel : comment doivent être les choses les « choses mêmes »- pour que ce que nous disons là soit vrai ou faux notamment étant entendu que les façons que nous avons de nous rapporter linguistiquement au réel ne se réduisent nullement à la simple modalité du vrai et du faux.

Maintenant, il est étrange de faire de cette réalité qui peut rendre nos énoncés respectivement vrais ou faux une réalité sur parole. De ce point de vue, la circularité apparente suivant laquelle ce n'est que la chose telle qu'on la dit qui peut rendre vrai ce qu'on en dit, pourrait bien se révéler un fort mauvais argument. En effet, elle soulève inévitablement la question de savoir sous quelles conditions on peut dire cette chose telle qu'on l'a dit: la question des conditions réelles de l'application d'une telle norme de référence.

En fait, en règle générale, le problème de l'idéalisme linguistique, y compris de l'idéalisme linguistique critique, est qu'il tend à couper le langage de sa propre réalité, donc de la réalité tout court, et à le représenter comme un empire dans un empire : comme si nos énoncés en quelque sorte flottaient indépendamment de toutes conditions d'énonciation. Un tel langage peut dès lors être représenté comme un sas ou un vestibule qui commanderait, médiatiserait et éventuellement occulterait notre accès à la réalité. Saurons-nous jamais comment sont les choses indépendamment du fait que nous en parlons?

Le problème de ce genre d'interrogation qui instaure pour ainsi dire une compétition entre l'être des choses telles qu'elles sont et l'être de ces mêmes choses telles qu'on en parle, est que, en un sens, elle ne peut que rendre ces deux êtres excessivement commensurables au moment même où elle affirme leur incommensurabilité. En effet, pointer un écart entre ces deux êtres, c'est à un certain niveau les traiter comme des choses du même genre. Comme si, pour ainsi dire il pouvait y avoir ce que nous disons de la chose et ce qu'elle dirait d'elle-même. Seulement voilà, la chose ne dit rien. Elle n'est jamais que ce qu'elle est. Et même si, dans certains cas particuliers, son être consiste en un dire - là où il s'agit de cette forme d'être que nous appelons « discours »-, alors il ne se dit pas. La chose est dite là où il y a quelqu'un pour la dire. Reste de toute façon une distinction catégoriale à faire entre l'être tout simplement et le dit de l'être. Précisément les choses ne se disent pas d'elles- 
mêmes : le fait qu'elles soient dites requiert que quelqu'un fasse l'effort de les dire. De ce point de vue, l'opposition entre la chose elle-même, d'un côté, et la chose telle qu'elle peut être dite, de l'autre, entendue d'une certaine façon, pourrait bien exprimer le fantasme d'un discours sur la chose «sans effort », c'est-à-dire qui n'aurait pas à faire l'effort d'être un discours: qui ferait l'économie de construire une prise particulière sur la chose, la capturant prétendument indépendamment de toute norme de discours, « dans sa réalité » pure et simple.

Cependant si, lorsque notre discours est efficace, c'est bien à la réalité qu'il se rapporte et le réel comme tel qu'il caractérise, précisément il le caractérise, donc le détermine, selon une certaine norme, et cerner ce réel, c'est ainsi nécessairement le déterminer. Il n'y a pas de façon de parler de la chose sans en parler d'un certain point de vue, cela non pas au sens d'un point de vue subjectif - comme si la chose était relative au locuteur - mais en celui d'un certain sens précisément. Que la chose ne s'identifie pas à ce sens ne constitue pas réellement un problème, mais est purement et simplement trivial : il ne faut pas, ici, confondre une différence de catégorie avec une différence de contenu. Ce n'est pas que le chose est différemment de la façon dont elle est représentée - si la représentation est correcte, analytiquement, elle capture la chose dans son être même : c'est là la définition d'une « représentation correcte ». C'est plutôt qu'être, pour la chose, demeure toujours catégorialement différent d'être représenté - à telle enseigne que, suivant le dit kantien, il ne suffit jamais d'être représenté pour être. De ce point de vue, il n'y a pas de sens positif à opposer l'être de la chose à son être dit ; aucun autre sens, en tout cas, que celui d'une délimitation catégoriale, mais certainement pas celui d'une possible différence au sein d'une même catégorie : comme si la chose réelle était une autre chose que la chose dite. Elle est tout simplement autre que le dire qui s'y rapporte, en tant que chose. Mais cette différence, loin de soustraire la chose au discours, est ce qui l'y livre là où, en tout cas, celui-ci a correctement aménagé sa prise sur le réel. Le fait qu'il ne fasse pas, en règle générale, partie du sens de la chose même d'être dite ne constitue pas un obstacle au fait qu'elle le soit, puisque c'est précisément cet être non discursif de la chose en tant que chose qui est en jeu dans le discours, et que celui-ci cerne avec plus ou moins de succès suivant les rencontres. Si le fait d'être dite ne fait pas, normalement, partie du sens de la chose, le fait de dire la chose-même (telle qu'elle est hors discours) fait au contraire partie du sens du discours au moins dans tout un certain type d'emploi qui en est central, et sans doute définitionnel - et peut-être, en un sens élargi, dans tous ses emplois. 
Qu'est-ce qui, cependant, encore une fois, empêchera le métaphysicien - ou ce négatif du métaphysicien, produit de la modernité, qu'est le philosophe transcendantal de soutenir que, justement, on ne peut jamais être sûr de ce que les choses soient réellement telles qu'elles apparaissent dans notre discours, y compris là où celui-ci satisfait toutes les normes d'objectivité qui sont, précisément, celles du discours - et seulement celles du discours?

On remarquera le point auquel cette inquiétude métaphysique - ou sa solution transcendantale : nous n'avons que les choses telles que nous les disons, mais elles nous suffisent car c'est là tout ce qu'il y a à dire - peut avoir partie liée avec cette mise en exergue de la certitude comme valeur absolue qui est constitutive de la modernité. Sommes nous bien sûrs que... ?

Il y aurait pourtant lieu de distinguer le savoir de la certitude : souvent, nous sommes certains de choses que nous ne savons pas; et inversement tout savoir ne se présente pas forcément sous la modalité de la certitude, même si l'incertitude, probablement, est incompatible avec un véritable savoir - l'erreur des modernes, ici comme souvent, est d'avoir pris la négation de la négation pour une affirmation. D'autre part, bien sûr, le rapport que nous avons aux choses dans leur réalité - c'est-à-dire leur être de choses - ne se réduit nullement au savoir. Pourquoi donc notre accès à leur réalité dépendrait-il si absolument de notre capacité à « les connaître telles qu'elles sont » ? Il est possible, de ce point de vue, que toute formulation purement épistémique de la question du « réalisme » - et, symétriquement, tout supposé constat d'échec sur ce terrain - ipso facto manque sa cible.

Le passage dans le registre linguistique éclaire de toute façon le problème d'un autre jour. D'abord, il n'est pas sûr que la notion d'apparaître ou de phénomène au sens où l'emploie l'argument demeure absolument pertinente là où il s'agit de discours. Il y aurait d'autres raisons de s'interroger sur sa pertinence là où il s'agit de perception ${ }^{5}$. Il n'est pas sûr du tout qu'il y ait le moindre sens à traiter la perception par principe comme une forme d' « apparaître », comme l'a largement fait la philosophie moderne. En fait, au moins dans sa version originaire, le jeu de langage de l'apparaître dépend de celui de la perception, et non l'inverse. C'est au sein du perçu, en règle générale, que, sous certaines conditions, cela a un sens de dire de certaines choses en particulier

\footnotetext{
${ }^{5}$ Voir Jocelyn Benoist, Le Bruit du Sensible, Paris, Hermann, coll. « Passages », 2013, et Logique du Phénomène, Paris, Hermann, coll. « Le Bel aujourd'hui », 2016.
} 
qu'elles « apparaissent» - par opposition à d'autres, qui «n'apparaissent pas », et en fonction de certaines attentes que nous plaçons ou nous ne plaçons pas dans le perçu.

Il reste que l'apparaître, précisément, constitue une dimension possible de la perception, comme lieu où se rencontrent apparences, reflets et autres aspects.

Ce que peut vouloir dire une « apparence linguistique », en tout cas au sens propre du terme, est moins clair. Bien sûr, sous certaines conditions, on peut s'exprimer dans ces termes : « à la lumière de ce que tu m'as dit, la situation m'est apparue sous un autre jour ». Il s'agit cependant d'une métaphore. Dans le langage, la chose n' " apparaît» pas, ou alors il s'agit là d'une spécificité du discours figuré, qui, précisément, a vocation à produire cette illusion d'apparaître, en conférant un statut sensible à ce dont il est parlé. Il pourrait aussi s'agir d'une définition de la rhétorique au sens classique du terme, dont l'idéal était de «mettre la chose sous nos yeux $\rangle^{6}$ par le discours. Il est alors néanmoins essentiel que la chose en question, précisément, ne soit rien d'autre qu'une chose de discours - que ce soit le discours qui, alors, définisse ce qu'il y a à voir - et la notion d'apparaître demeure alors métaphorique : il s'agit d'un apparaître, comme on dit, « devant les yeux de l'esprit».

Tout discours ne répond de toute façon pas à l'idéal rhétorique de l'hypotypose. Et s'il est possible, parfois, que le discours, par des procédés déterminés, s'arroge apparemment la puissance de «faire apparaître » quelque chose - ou en tout cas nous propose de jouer à ce jeu - il ne s'agit que d'une mise en œuvre des mots parmi d'autres et qui, loin de le constituer, suppose le rapport fondamental de ces mots à la réalité, qui ne consiste pas spécialement à la « faire apparaître » mais, de façons du reste multiples et variées, et à différentes fins, à s'y référer. Référer à une chose ce n'est pas, spécialement, la faire apparaître, mais pour ainsi dire exercer une prise sur la réalité. Et, en référant aux choses, c'est de leur côté et non du côté des «phénomènes » que nous renvoie notre interlocuteur, de telle façon que nous soyons en position, précisément, si c'est nécessaire, de dénoncer ce qu'il nous raconte comme " pure apparence ». Même le discours de l'apparaître - « regarde, vu ainsi, cela a tel ou tel air » - suppose un tel engagement par rapport au réel, un engagement que l'interlocuteur prend par rapport à moi : en disant des choses « qu'elles ont tel ou tel air », ou « qu'on est confronté à telle ou telle apparence », c'est par rapport au réel encore qu'on prend position - ou que, dans le second cas, on refuse de prendre position, mais refuser de prendre position par

${ }^{6} \mathrm{C}$ 'est en ce sens que la rhétorique classique était fondamentalement «phénoménologique », comme l'a bien montré Thibaut Sallenave dans : Le phénomène topique. Phénoménologie, grammaire et rhétorique du lieu commun, thèse soutenue à l'université Paris 1 Panthéon-Sorbonne le 12 décembre 2014. 
rapport à quelque chose, c'est, en un sens, encore avouer notre appartenance à la réalité : on ne dénie pas alors le réel comme tel, mais on ne sait tout simplement pas bien s'il faut y placer une certaine chose. C'est qu'on ne peut « dire » sans mordre d'une certaine façon sur le réel. C'est au réel, fondamentalement, que sont ordonnés les mots et ce que nous appelons ordinairement « langage », y compris dans leur façon parfois de se mettre en vacances par rapport à lui - on ne se met en vacances, évidemment, que de son métier et de sa vocation ordinaires.

Le langage, en ce sens, loin de constituer en soi une nouvelle sphère d'apparaître, est ce qui, constitutivement, nous place au-delà de l'apparaître. Cela, non pas au sens où il en risquerait l'hypothèse, ou, pour reprendre les termes kantiens, il « dépasserait le donné », sans que sa légitimité à le dépasser soit bien assurée. Certes, nous disons toujours plus qu'il ne nous est donné. Mais c'est que ce qui, du point de vue d'une pensée qui fait du donné sa norme, est conçu comme un « excédent», constitue en fait bien plutôt la norme du donné. C'est en vertu de l'appariement originaire de nos mots avec les choses - et il faut entendre par là «les choses mêmes », « la réalité » - que nous pouvons, sous certaines conditions, les considérer comme «données » ou non. Cette notion, cependant, n'a de sens que parce que nous sommes déjà en contact avec les choses et, si étrange que cela puisse sonner aux oreilles des modernes métaphysiciens, qui sont en règle générale des métaphysiciens empiristes donc des métaphysiciens retournés (mais non moins métaphysiciens pour autant), c'est par le langage que nous le sommes. Cela tout simplement parce que ce que nous nommons « langage » ne renvoie à rien d'autre qu'à la fixation de normes sur la réalité de la chose, qui permettent d'appréhender cette réalité-même de certaines façons et, d'autant de façons différentes, de la caractériser. C'est dans cet espace normatif qui est celui du langage que se joue notre rencontre avec le réel. Non pas, évidemment, qu'en-deçà du langage il n'y ait pas de réel ; mais simplement, dans cet en-deçà (qui est un en-deçà logique, et non métaphysique), le réel est tout simplement ce qu'il est, et nous en faisons partie. Partout où nous voulons l'appréhender d'une certaine façon et le caractériser, il faut une norme, et ce qu'on appelle « langage » n'est rien d'autre que le répertoire - fixé dans l'usage - de ces normes.

Ce n'est pas un pari sur le réel que fait le langage. C'est une prise normative qu'il exerce sur lui, plus ou moins bien - dans certains cas, nous croyons parler alors qu'en fait nous ne disons rien. Or une telle prise, en un certain sens, est elle-même réelle, et a des conditions réelles. On parle dans la réalité de la réalité. 
De ce point de vue, la modulation en vertu de laquelle, dans le deuxième temps de Dire le Monde, on passe de la thèse suivant laquelle « le langage fait monde » à celle suivant laquelle «la parole fait monde » pourrait bien s'avérer plus que la simple transposition ou contrepartie d'un même thème. En réalité, la seconde formule délivre la vérité de la première.

En effet, « le langage », là où personne ne parle, ne fait rien, et moins que rien un monde. Seul peut le croire un relativisme linguistique qui traite le langage, sous l'espèce de « la langue », précisément comme un monde - autant de langues, autant de mondes, dit-on, comme si tous ces mondes se trouvaient déjà pour ainsi dire en pièces détachées dans les langues en question, et s'il ne restait plus aux locuteurs qu'à les monter. Or les mots, par eux-mêmes, ne disent rien ${ }^{7}$. C'est nous qui disons avec eux. Et, que ce que nous disons, nous le disions toujours avec les moyens expressifs dont nous disposons qui sont, par définition, ceux d'une langue - n'a jamais suffi à en prédéterminer le contenu, ni même, sans doute, en un certain sens à le limiter. Si une langue est vraiment une langue, elle supporte, par définition, que l'on dise avec elle des choses que, comme on dit, elle n'a pas été faite pour dire, au sens où ces choses ne font pas partie du répertoire des choses habituellement dites avec elle et qui donc ont contribué à fixer ses structures. En fait, en toute rigueur, tout ce dont on peut, dans les termes d'une certaine langue, se poser la question de le dire est, dans les termes mêmes de cette langue, dicible. En ce sens-là, ce n'est en aucun cas la langue qui constitue la forme de mon monde. Cette thèse, séduisante par l'espèce d'irresponsabilité dans laquelle elle nous installe, est en elle-même dépourvue de sens.

En revanche, il est certainement incontestable que la réalité à laquelle nous avons affaire révèle son aspect déterminé dans ce que, dans certaines circonstances, nous en dirions - se révèle "comme nous le dirions alors ». En ce sens-là, le langage, assurément, est indicateur de monde : dans sa sensibilité à la réalité - c'est-à-dire : dans la sensibilité à la réalité de la norme qu'il définit sur elle - il en débusque les traits.

Seulement, ce dont il s'agit alors n'est plus la langue, mais la parole : c'est elle, en tant qu'elle se plie aux règles des jeux qui la définissent (comme telle ou telle), qui peuple le monde, ou en tout cas en fait ressortir le grain et les traits. Si parler c'est toujours, en un sens ou un autre, se prononcer sur les choses ou les mettre en jeu, il y a bien une dimension mondanéisante de la parole. On aurait presque envie de parodier

\footnotetext{
${ }^{7}$ De ce point de vue, nous ne pouvons souscrire au principe de la distinction entre la façon dont «le langage » et « la parole » seraient censés respectivement « faire monde » avancé dans DM, p. 195. En effet, « le langage », en lui-même, ne « dit » rien, pas plus localement que « globalement ».
} 
Wittgenstein, en disant : «Ce qui est réel, c'est ce que les hommes disent »- cela non pas au sens d'une réduction du réel à ce qui est dit - le réel n'a pas besoin d'être dit pour être réel - mais en celui où c'est dans cela même que « les hommes disent » (dans la variété de ce qu'ils disent) que s'exprime la réalité du réel.

Cependant, il n'y a de parole, précisément, que dans le réel. D’abord parce que la parole est un acte et qu'il n'y a d'action, fût-ce celle de parler, que réelle. Entre tout rêve de faire et l'action subsiste toujours un gouffre : celui de l'effectuation. Encore une fois : on ne peut pas parler sans le faire, et le faire, c'est le faire effectivement. Ensuite, parce que, comme toute action, cet acte a des conditions réelles.

Il ne s'agit pas ici des conditions externes qui peuvent peser sur l'acte, c'est-à-dire du réseau causal dans lequel il se trouve nécessairement pris en tant qu'événement réel : le fait que je dise ce que je dis parce que je suis qui je suis, mon éducation m'y a déterminé, etc. etc. Il s'agit bien plutôt des conditions normatives qui déterminent la possibilité - la possibilité logique, devra-t-on dire - de chaque acte de langage pris dans sa spécificité. Un acte de langage définit toujours - c'est le principe même de l'illocutionnaire, qui se caractérise par ses effets conventionnels, par opposition au perlocutionnaire, qui dépend essentiellement de sa capacité à produire des effets non conventionnels, donc réels - une certaine norme sur le monde, norme selon laquelle la réalité est déterminée et, en un sens, révélée : ici, je tiens ma promesse, là je ne la tiens pas; ici, la situation est bien telle que je l'énonce, là, elle ne l'est pas. Toutefois, le même acte de langage requiert aussi, logiquement, que certaines conditions soient réalisées, afin qu'il ait son sens et qu'il puisse être effectué. Ainsi ne puis-je par exemple te faire don que de ce qui est à moi ou te promettre que ce qu'il y a des raisons de penser - et ce que je pense - être en mon pouvoir, ou en tout cas tel que cela puisse le devenir. Autrement, mon acte change purement et simplement de nature: d'une promesse, cela devient un encouragement, ou une bonne parole.

La nuance entre « ce qu'il y a des raisons de penser» et « ce que je pense », ici, est particulièrement intéressante. En effet, il ne suffit pas, pour que ma promesse en soit une, que je croie que ce que je promets est réalisable - que j'aie l'intention de tenir ou non cette promesse, du reste; le problème de savoir si une « fausse promesse » est une promesse est en substance encore une autre question. Il faut que cela le soit. Quelqu'un peut me dire à bon droit: «vous ne pouvez pas promettre cela: en effet, cette chose n'est pas en votre pouvoir. » L'impossibilité ainsi formulée est logique - celle d'une contradiction avec le sens même de l'acte qui consiste à promettre - et non physique, 
même si elle s'adosse éventuellement à une impossibilité physique : celle, pour moi, de faire une certaine autre chose.

La prise en compte de la parole nous situe donc de plain pied dans le réel : celui-ci se trouve, pour ainsi dire, en amont et en aval d'elle. Le caractère d'acte du langage contraint le philosophe, pour peu qu'il prête un peu attention à ses conditions, au réalisme. Transportée sur ce terrain, en effet, la logique - en tant que théorie du logos, du discours - touche au réel.

En ce sens, s'il est bien vrai que « la parole fait monde » - puisqu'elle est la façon même pour nous d'assigner aux choses auxquelles nous sommes confrontés une place dans le monde - elle ne peut pas le faire au sens où ce qui, du langage, ne serait pas parole, le ferait - au sens où donc, d'après la première partie du livre, « le langage » tout simplement « faisait monde ». Il ne s'agit pas de deux vues complémentaires sur le monde - le monde sans agent versus le monde avec agent, en quelque sorte -, fussentelles inextricablement entrelacées. Seule la ou plutôt les paroles tiennent compte tiennent les comptes - du monde, cela parce qu'elles sont situées dans le réel d'une façon définie, et que, de cette façon, sur ce réel, elles construisent des prises définies.

De ce point de vue, on peut penser que, si une des vertus de l'analytique du langage et du monde - des différents « langages-mondes », ainsi qu'il se plaît à le dire déployée par Francis dans Dire le Monde est d'avoir bien mis en avant l'importance et l'irréductibilité de la parole, il reste peut-être quelque chose d'égarant dans le modèle fondamentalement benvenistien appliqué à la parole. Si Francis trouve bien dans la tradition du linguiste français les ressources d'une pensée de l'énonciation, il s'agit alors de l'énonciation inscrite en langue, suivant «l'appareil formel de l'énonciation », qui se traduit notamment par le jeu des pronoms. Une telle perspective tend, en faisant de l'acte même, et donc de la parole, un phénomène de langue - comme une dimension personnelle de la langue, qu'on aurait laissée de côté en un premier temps pour s'étonner par après de la retrouver - à isoler excessivement la parole du réel, en la plaçant dans une forme d'autonomie qu'elle partagerait avec la langue, même si elle vient en corriger le caractère impersonnel.

Or l'énonciation, avant d'être affaire de personnes, est d'abord affaire de position: le locuteur ne dit exactement ce qu'il dit qu'en vertu de sa situation dans le réel, comme l'a profondément diagnostiqué Austin dans son texte fondateur sur la vérité $^{8}$. De la même façon, s'il faut parler d'action - Francis fait de la possibilité de dire

\footnotetext{
${ }^{8}$ John Langshaw Austin, « Truth » (1950), traduction française Lou Aubert et Anne-Lise Hacker : « La
} 
«Je » le fondement même de ce qu'il appelle «action »- il faut alors souligner qu'il n'y a pas d'agentivité sans conditions réelles : le réel est en amont de l'acte, qui suppose toujours une façon de s'y disposer et d'en jouer, et non seulement la matière à quoi il s'applique - de façon, peut-être, à « en faire un monde ».

On l'aura compris, ces remarques plaident pour un réalisme peut-être plus dispendieux - plus réellement engagé, et autant embarqué qu'engagé - que l'idéalisme transcendantal linguisticisé défendu, en première approche, dans Dire le Monde. Faire passer l'analyse sur le terrain du langage, c'est, nous semble-t-il, la forcer à sortir de sa neutralité de principe (quant à la question du réalisme ou de l'idéalisme).

Toutefois, il se pourrait que le kantisme quelque peu académique affiché par Dire le Monde, suivant une synthèse harmonieuse de la philosophie contemporaine (nous voulons dire : du langage) et de la philosophie moderne, ne constitue que la surface du livre et que l'auteur lui-même ne s'en tienne précisément qu'en première approche à cette «neutralité ». Tout au long de l'enquête menée, un élément essentiel, autour duquel pivote l'analyse depuis le début, est présent qui en effet rompt la neutralité. Or, cela, Francis le doit au fait que, comme la préface de l'édition «Quadrige » le précise, avec la clarté sur ses intentions et sur sa propre position énonciative qui caractérise une démarche philosophique radicale, la réflexion sur le langage présentée dans cet essai s'enracine bien plutôt dans la philosophie ancienne - cette philosophie que Francis a toujours cultivée et aimée et dans laquelle il se sent pour ainsi dire chez soi - que dans la philosophie contemporaine. On ajoutera même, d'une façon qui déplaira sans doute à l'auteur car, comme toute une génération, il est persuadé d'avoir joué avec bonheur Aristote, supposé plus près de notre empirisme de Modernes, contre Platon : dans un paradigme platonicien, parce que dialogique, de la philosophie. S'il est possible, comme le fait Francis, d'appliquer un tel paradigme à Aristote et de proposer une interprétation dialogique du principe de contradiction et de la prédication - ce qui constitue un des deux grands résultats de ce livre (l'autre ayant trait au jeu de langage de l'action, ou à cette dimension du langage qu'est l'existence linguistique de l'agent) - c'est parce que, fondamentalement, Aristote est un platonicien, même si un platonicien dissident. La philosophie ancienne, plus que celle d'aujourd'hui, était structurée en écoles parce qu'il faisait partie du concept de «philosophie» tel qu'il avait été instauré précisément par Platon qu'on ne fasse pas de la philosophie tout seul.

vérité », dans John Langshaw Austin, Écrits Philosophiques, Paris, Seuil, coll. « La couleur des idées », 1994. 
Dans la conférence donnée en 2003 à la Société française de philosophie reproduite en annexe (Annexe 2) de l'édition Quadrige, «L'impossible, l'interdit et le monde commun », Francis revient sur la question de l'idéalisme par un biais qui éclaire d'un autre jour la ligne argumentative développée dans Dire le monde. Il met alors en avant la transformation que fait subir au problème le fait de le transporter sur le terrain du langage :

Résoudre le rapport du langage au réel n'est donc jamais (ou du moins ne devrait jamais revenir à) se demander comment s'articulent deux entités face a face - le langage d'un côté, le réel de l'autre - mais comment il est possible à quelqu'un (qui que ce soit) de parler à quelque autre (qui que ce soit) de quelque chose (quoi que ce soit). (DM p. 272273)

C'est que, comme le précise la préface de l'édition Quadrige :

L'expression même de «mon langage » est une contradiction dans les termes - du moins si le langage est logos, c'est-à-dire à la fois un « parler à » et un «parler de ». $(D M 11)^{9}$

Parler, c'est toujours déjà s'orienter dans un espace où l'on n'est pas tout seul, qui est espace logique. Francis distingue parfois « logique » et « dialogique », comme si la seconde dimension ajoutait quelque chose à la première, ou si la première pouvait subsister indépendamment de la seconde, mais on peut en douter: qu'est-ce que la logique, étymologiquement, si ce n'est ce qui nous permet de nous rendre les uns aux autres des comptes, logon didonai ? Il faudrait, ici comme ailleurs, réveiller le sens originaire de la logique comme dialectique ${ }^{10}$ - cette dialectique des Anciens sous l'horizon théorique de laquelle Francis inscrit résolument sa démarche.

Une fois qu'on a rendu sa dimension d'adresse et de responsabilité au discours, la question du « réalisme » se pose bien différemment car, du fait même de parler, nous

\footnotetext{
${ }^{9}$ Il est évidemment piquant que l'objection, ici, soit adressée à Wittgenstein - «les limites de mon langage signifient les limites de mon monde »(Tractatus, 5.6). En effet, en un certain sens, dans sa dimension d'autocritique, l'argument du « langage privé » - c'est-à-dire de l'impossibilité constitutive de tout «langage privé » - introduit par la seconde philosophie, est largement convergent avec le point critique, et radicalement philosophique, au sens platonicien du terme, mis en avant par Francis. En tout cas, à l'arrière-plan des deux démarches, se trouve certainement un seul et même problème. Pour le Wittgenstein de la maturité aussi parler de "mon langage » en ce sens absolu - je peux utiliser des idiolectes ou avoir un style mais c'est une autre question - est une contradiction dans les termes. Ce qui, comme pour Francis, n'ôte rien au fait que, là où quelque chose m'est demandé, c'est moi et moi seul qui ai à parler et que personne ne peut le faire à ma place.

${ }^{10} \mathrm{Il}$ faudrait penser ici à l'usage fait du terme par Jean-Yves Girard, dans certaines phases de son œuvre, pour caractériser la logique même, ou à la notion de «dispute » telle qu'on la trouve au centre de cette logique qu'il a baptisée « ludique ». Un trait général des nouvelles logiques qu'il a inventées, suivant le mouvement d'expérimentation le plus créatif de la fin $\mathrm{du} \mathrm{XX}^{\mathrm{e}}$ siècle en logique, est qu'il n'y a de « logique », pour lui, que là où d'une certaine façon, se construit quelque chose comme une interaction réglée. Francis fait du reste lui-même un pas dans le sens de la caractérisation de la logique elle-même comme « dialogique » en se référant à Lorenzen ( $D M$ p. 42-43).
} 
sommes toujours déjà dehors, auprès de ces choses dont nous avons à rendre compte à notre interlocuteur et sur lesquelles, essentiellement, nous pouvons disputer. En toute rigueur, nous passons notre temps à le faire : parler, c'est essentiellement requalifier les choses par rapport à ce qu'en dit l'interlocuteur et, réciproquement, s'exposer soi-même au risque d'une telle requalification, qui requerra de notre part, si nous tenons à la qualification que nous avons avancée, que nous donnions des raisons : des raisons dans lesquelles l'autre peut rentrer, c'est-à-dire aussi qu'il peut discuter.

Ainsi se comprend l'affirmation fondamentale de la préface de l'édition Quadrige, un peu surprenante par rapport aux accents kantiens de l'édition de 1997 :

Nous avons ainsi voulu renouer avec la source même du "réalisme" des Anciens. (DM, p. 11)

Inutile de dire la vive sympathie que suscite en nous une telle affirmation. Un réalisme fondé dans la présence du logos (dans lequel l'idéaliste, par construction, n'est pas « présent »), qui est présence à l'autre, et non présence solitaire, quel plus magistral, parce que plus simple, plus primordial retour de réel peut-on rêver? Non point celui des bâtisseurs de monde, enivrés de leurs constructions solipsistes du droit d'une raison monologique - ce que nous nommons, pour notre part, «ontologie », qui en fait nous éloigne du réel, y substituant sa représentation (une « image du monde ») - mais celui qui, dans des rencontres données, peut venir concrètement en débat des uns aux autres.

La question qu'il resterait alors sans doute à poser est de savoir si une telle perspective peut encore être qualifiée de «transcendantale», comme Francis semble tenir à le faire. Certes, dans la préface de 2004, on trouve cette mise en garde précieuse - qui, peut-être, n'est pas sans avoir une portée corrective par rapport à l'impression qui aurait pu résulter de la première édition :

Cette définition d'un langage, déterminé par ces deux relations indissociables (que nous appelons respectivement « relation d'objectivité » et « relation d'interlocutivité ») est le meilleur rempart contre toute forme d'idéalisme théorique - même contre ce nouvel « idéalisme transcendantal» dans lequel les schèmes du langage seraient mis à la place des conditions a priori de la connaissance. (DM, p. 10)

Et de façon décisive, l'auteur en conclut, quelques lignes plus loin : «Il devient donc absolument inutile de réfuter l'idéalisme. »

C'est dire que le spectre de l'analyse transcendantale semble s'éloigner, car en quoi d'autre celle-ci pourrait-elle consister qu'en une telle «réfutation » - qu'en l'établissement de la possibilité de l'accès au monde en tant que « réel » ? Telle serait la portée d'une telle analyse, en tout cas, si nous en croyons cette lecture de la Déduction 
kantienne qui consiste à en faire une réponse au défi du scepticisme - réponse qui passe, tout d'abord, par une reformulation de ce défi ${ }^{11}$.

S'installer sur le terrain du dialogue, là où nous nous parlons les uns aux autres et où nous disputons des choses, c'est en effet se placer en un point où le problème du rapport du langage au monde ne peut pas se poser parce que, en un certain sens, le langage l'a toujours déjà résolu ${ }^{12}$. Ou plutôt, le métaphysicien ne peut se figurer qu'il y ait ici un problème à résoudre, et en faire du langage la «solution », que parce que, justement, il croit qu'il y a un sens, du point de vue théorique, pour poser la question qui l'intéresse, à se placer en position d'extériorité par rapport au langage, à se retirer en quelque sorte du dialogue, et à interroger de l'extérieur, absolument, l'appariement des mots avec les choses. Le langage apparaît alors en fait artificiellement, et de façon en toute rigueur absurde, comme une solution à un problème qui, là où on l'utilise, c'est-àdire où il est langage, ne se pose pas.

L'adoption d'une perspective véritablement linguistique, où le langage est le langage réel, en acte, semble donc désamorcer toute interrogation de type transcendantal, y coupant court, pour ainsi dire à la racine. La question de la possibilité pour nous de mettre en œuvre des normes ne se pose pas, car nous en appliquons déjà toujours et nous disputons toujours déjà sur leur application : qu'est-ce qui fait norme, et quelle norme faut-il appliquer et comment? Telles sont les questions que soulèvent couramment nos façons de parler : il n'est pas un acte de langage qui ne constitue, dans son genre propre, un pas dans un espace normatif, où il y va du réel et de la correction de notre rapport au réel, cela sur un mode public et soumis à une possible discussion par autrui. La norme, ici, n'a pas à être gagnée depuis un néant de norme, et elle nous renvoie immédiatement - mais pas pour cela de façon moins disputée, au contraire - au réel.

Pourtant, il semblerait que, dans son dernier temps, le livre réassume un point de vue de type transcendantal, dans sa mise en avant du principe qu'il nomme « allocution transcendantale ». Comme si, après avoir « déduit» que toute parole est parole d'un « Je », il fallait « déduire » qu'elle est adressée. De ce point de vue, tout dépend de la façon dont il faut entendre le verbe « supposer » dans la phrase suivante :

Toute parole suppose quelqu'un qui parle, mais aussi un autre à qui il parle. (DM, p. 206)

\footnotetext{
${ }^{11}$ Pour une telle lecture, voir James Conant, « Varieties of Skepticism », dans Denis McManus (éd.), Wittgenstein and Scepticism, Londres, Routledge, 2004, p. 97-136.

${ }^{12}$ «In der Sprache wird alles ausgetragen», comme dirait Wittgenstein, Philosophische Grammatik, I, $\S 95$, dans Werkausgabe, t. IV, Suhrkamp, 1984, p. 143.
} 
S'il s'agit d'une exigence - qui pourrait, en principe, ne pas être satisfaite, et dont la satisfaction rend seulement (positivement) possible que la «parole » en question en soit une, comme s'il y avait pour elle une façon d'en être une à moitié, d'en être une sans en être une, en amont et ou en attente de cette satisfaction - alors, nous avons affaire à une démarche philosophique de type transcendantal. Ce sens positif de l'impossibilité et, symétriquement, de la possibilisation (du « rendre possible ») en est en effet la marque la plus sûre. Il suppose que le philosophe, pour ainsi dire, continue de croire possible de - ou en tout cas s'autorise à, même s'il le croit impossible - avoir un pied dans et un pied en dehors du langage (et de la réalité).

S'il s'agit d'une simple analyse : du pur et simple déploiement de ce que veut dire « parler»- c'est trivialement que « parler », c'est « parler à »- alors, on ne trouvera rien là à redire, au contraire: on aurait, dès lors, tout simplement affaire à une philosophie qui aurait tiré jusqu'au bout les conséquences du fait que nous (nous) parlons.

Mais c'est alors tout aussi trivialement que nous sommes au monde.

\section{Bibliographie}

Austin John Langshaw, " Truth» (1950), traduction française Lou Aubert et Anne-Lise Hacker: "La vérité », dans John Langshaw Austin, Écrits Philosophiques, Paris, Seuil, coll. « La couleur des idées », 1994.

Benoist Jocelyn, Le Bruit du Sensible, Paris, Hermann, coll. « Passages », 2013.

Benoist Jocelyn, Logique du Phénomène, Paris, Hermann, coll. « Le Bel aujourd'hui », 2016.

Conant James, «Varieties of Skepticism», dans Denis McManus (éd.), Wittgenstein and Scepticism, Londres, Routledge, 2004, p. 97-136.

Ehrsam Raphaël, Le problème du langage chez Kant, Paris, Vrin, coll. « Bibliothèque d'histoire de la philosophie », 2016.

Sallenave Thibaut, Le phénomène topique. Phénoménologie, grammaire et rhétorique du lieu commun, thèse sous la direction de Jocelyn Benoist, soutenue à l'université Paris 1 PanthéonSorbonne le 12 décembre 2014.

Wittgenstein Ludwig, Philosophische Grammatik. Werkausgabe, t. IV, hrsg. von Rush Rhees, Frankfurt am Main, Suhrkamp, 1984.

Wolff Francis, Dire le monde, Paris, Presses universitaires de France, 1997. 